hep-ph/0109083

\title{
Impact of bosonic decays on the search for $\tilde{t}_{1}$ and $\tilde{b}_{1}$ squarks
}

\author{
K. Hidaka \\ Department of Physics, Tokyo Gakugei University, Koganei, Tokyo 184-8501, Japan \\ E-mail: hidaka@u-gakugei.ac.jp
}

\begin{abstract}
We show that the bosonic decays of the lighter top and bottom squarks, i.e. $\tilde{t}_{1} \rightarrow \tilde{b}_{1}+\left(H^{+}\right.$or $\left.W^{+}\right)$and $\tilde{b}_{1} \rightarrow \tilde{t}_{1}+\left(H^{-}\right.$or $\left.W^{-}\right)$, can be dominant in a wide range of the MSSM parameters. Compared to the fermionic decays, such as $\tilde{t}_{1} \rightarrow b+\tilde{\chi}_{j}^{+}$, these bosonic decays can have significantly different decay distributions. We also show that the effect of the supersymmetric QCD running of the quark and squark parameters on the $\tilde{t}_{1}$ and $\tilde{b}_{1}$ decay branching ratios is quite dramatic. These could have an important impact on the search for $\tilde{t}_{1}$ and $\tilde{b}_{1}$ and the determination of the MSSM parameters at future colliders.

(Invited talk at The 9th International Conference on Supersymmetry and Unification of Fundamental Interactions (SUSY'01), 11-17 June 2001, Dubna, Russia; to be published in the Proceedings (World Scientific Pub.))
\end{abstract}

We study the decays of the lighter top and bottom squarks (i.e. $\tilde{t}_{1}$ and $\tilde{b}_{1}$ ) in the MSSM. They can decay into fermions, i.e. a quark plus a gluino $(\tilde{g})$, neutralino $\left(\tilde{\chi}_{i}^{0}\right)$ or chargino $\left(\tilde{\chi}_{j}^{ \pm}\right)$. They can also decay into bosons [1]:

$$
\tilde{t}_{1} \rightarrow \tilde{b}_{1}+\left(H^{+} \text {or } W^{+}\right), \quad \tilde{b}_{1} \rightarrow \tilde{t}_{1}+\left(H^{-} \text {or } W^{-}\right) .
$$

In case the mass difference between $\tilde{t}_{1}$ and $\tilde{b}_{1}$ is sufficiently large [2], the decays of Eq. (11) are possible. Here we extend the analysis of [四].

The squark mass matrix in the basis $\left(\tilde{q}_{L}, \tilde{q}_{R}\right)$ with $\tilde{q}=\tilde{t}$ or $\tilde{b}$ is given by [四]

$$
\begin{gathered}
\mathcal{M}_{\tilde{q}}^{2}=\left(\begin{array}{cc}
m_{\tilde{q}_{L}}^{2} & a_{q} m_{q} \\
a_{q} m_{q} & m_{\tilde{q}_{R}}^{2}
\end{array}\right) \\
m_{\tilde{q}_{L}}^{2}=M_{\tilde{Q}}^{2}+m_{Z}^{2} \cos 2 \beta\left(I_{3}^{q_{L}}-e_{q} \sin ^{2} \theta_{W}\right)+m_{q}^{2} \\
m_{\tilde{q}_{R}}^{2}=M_{\{\tilde{U}, \tilde{D}\}}^{2}+m_{Z}^{2} \cos 2 \beta e_{q} \sin ^{2} \theta_{W}+m_{q}^{2} \\
a_{q} m_{q}= \begin{cases}\left(A_{t}-\mu \cot \beta\right) m_{t} & (\tilde{q}=\tilde{t}) \\
\left(A_{b}-\mu \tan \beta\right) m_{b} & (\tilde{q}=\tilde{b}) .\end{cases}
\end{gathered}
$$


We treat the soft SUSY-breaking parameters $M_{\tilde{Q}, \tilde{U}, \tilde{D}}$ and $A_{t, b}$ as free ones since the ratios $M_{\tilde{U}} / M_{\tilde{Q}}, M_{\tilde{D}} / M_{\tilde{Q}}$ and $A_{t} / A_{b}$ are highly model-dependent. By diagonalizing the matrix (2) one gets the mass eigenstate $\tilde{q}_{1}=\tilde{q}_{L} \cos \theta_{\tilde{q}}+\tilde{q}_{R} \sin \theta_{\tilde{q}}$.

We take $M^{\prime}=(5 / 3) \tan ^{2} \theta_{W} M$ and $m_{\tilde{g}}=\left(\alpha_{s}\left(m_{\tilde{g}}\right) / \alpha_{2}\right) M$ with $M, M^{\prime}$ and $m_{\tilde{g}}$ being the $\mathrm{SU}(2), \mathrm{U}(1)$ gaugino and gluino mass, respectively. We denote the mass of the CP-odd Higgs boson $A^{0}$ as $m_{A}$. Full expressions of the widths of the squark decays are given in [1].

In case $M_{\tilde{Q}, \tilde{U}, \tilde{D}}$ are relatively large in Eqs.(2-5), for $M_{\tilde{U}}>M_{\tilde{Q}} \gg M_{\tilde{D}}\left(M_{\tilde{D}}>M_{\tilde{Q}} \gg\right.$ $\left.M_{\tilde{U}}\right)$ we have $m_{\tilde{t}_{1}} \gg m_{\tilde{b}_{1}}\left(m_{\tilde{b}_{1}} \gg m_{\tilde{t}_{1}}\right)$, which may allow the bosonic decays of Eq.(11). We consider two patterns of the squark mass spectrum: $m_{\tilde{t}_{1}} \gg m_{\tilde{b}_{1}}$ with $\left(\tilde{t}_{1}, \tilde{b}_{1}\right) \sim\left(\tilde{t}_{L}, \tilde{b}_{R}\right)$ for $M_{\tilde{U}} \gg M_{\tilde{Q}} \gg M_{\tilde{D}}$, and $m_{\tilde{b}_{1}} \gg m_{\tilde{t}_{1}}$ with $\left(\tilde{t}_{1}, \tilde{b}_{1}\right) \sim\left(\tilde{t}_{R}, \tilde{b}_{L}\right)$ for $M_{\tilde{D}} \gg M_{\tilde{Q}} \gg M_{\tilde{U}}$. Thus the bosonic decays considered here are basically the decays of $\tilde{t}_{L}$ into $\tilde{b}_{R}$ and $\tilde{b}_{L}$ into $\tilde{t}_{R}$.

The leading terms of the squark couplings to $H^{ \pm}$are given by

$$
\begin{aligned}
G\left(\tilde{t}_{1} \tilde{b}_{1} H^{ \pm}\right) \sim & h_{t}\left(\mu \sin \beta+A_{t} \cos \beta\right) \sin \theta_{\tilde{t}} \cos \theta_{\tilde{b}} \\
& +h_{b}\left(\mu \cos \beta+A_{b} \sin \beta\right) \cos \theta_{\tilde{t}} \sin \theta_{\tilde{b}} .
\end{aligned}
$$

The Higgs bosons $H^{ \pm}$couple mainly to $\tilde{q}_{L} \tilde{q}_{R}^{\prime}$ combinations. These couplings are proportional to the Yukawa couplings $h_{t, b}$ and the squark mixing parameters $A_{t, b}$ and $\mu$ ( Eq.(6)). Hence the widths of the squark decays into $H^{ \pm}$may be large for large $A_{t, b}$ and $\mu$. In contrast, the gauge bosons $W^{ \pm}$couple only to $\tilde{q}_{L} \tilde{q}_{L}^{\prime}$, which results in suppression of the decays into $W^{ \pm}$. However, this suppression is largely compensated by a large extra factor steming from the contribution of the longitudinally polarized $W$ boson radiation $\left(\tilde{q}_{1} \rightarrow \tilde{q}_{1}^{\prime} W_{L}^{ \pm}\right)$. Hence the widths of the squark decays into $W^{ \pm}$may be large for a sizable $\tilde{q}_{L}^{\prime}-\tilde{q}_{R}^{\prime}$ mixing term $a_{q^{\prime}} m_{q^{\prime}}$. On the other hand, the fermionic decays are not enhanced for large $A_{t, b}$ and $\mu$. Therefore the branching ratios of the bosonic decays of Eq.(11) are expected to be large for large $A_{t, b}$ and $\mu$ if the gluino mode is kinematically forbidden.

The widths of the $\tilde{t}_{1}$ and $\tilde{b}_{1}$ decays into $H^{ \pm}$receive very large SUSY-QCD corrections for large $\tan \beta$ in the on-shell (OS) renormalization scheme [4], making the perturbative calculation unreliable. This problem can be solved by carefully defining the relevant tree-level couplings in terms of appropriate running parameters and on-shell squark mixing angles $\theta_{\tilde{q}}$ [5]. Following Ref. [5], we calculate the tree-level widths of the squark decays by using the corresponding tree-level couplings defined in terms of the SUSY-QCD running parameters $m_{q}(Q)$ and $A_{q}(Q)$ (with $\mathrm{Q}=$ (on-shell mass of the decaying squark $\left.m_{\tilde{q}_{1} O S}\right)$ ), and the on-shell squark mixing angles $\theta_{\tilde{q}}$. We call the widths thus obtained as 'renormalization group (RG) improved tree-level widths'. Our input parameters are all on-shell ones except $A_{b}$ which is a running one, i.e. they are $M_{t}, M_{b}, M_{\tilde{Q}}(\tilde{t}), M_{\tilde{U}}, M_{\tilde{D}}, A_{b}\left(Q=m_{\tilde{q}_{1} O S}\right), A_{t}, \mu, \tan \beta, m_{A}$, and M. $M_{\tilde{Q}}(\tilde{q})$ is the on-shell $M_{\tilde{Q}}$ for the $\tilde{q}$ sector. The procedure for getting all necessary on-shell and SUSY-QCD running parameters is given in [5]. For the calculation of the Standard Model running quark mass $m_{q}(Q)_{S M}$ from the two-loop RG equations we use the two-loop running $\alpha_{s}(Q)$ as in [5]. We take $M_{t}=175 \mathrm{GeV}$ and $M_{b}=5 \mathrm{GeV}$. We choose $M_{\tilde{Q}}(\tilde{t})=\frac{3}{4} M_{\tilde{U}}=\frac{3}{2} M_{\tilde{D}}\left(M_{\tilde{Q}}(\tilde{t})\right.$ 
$\left.=\frac{3}{2} M_{\tilde{U}}=\frac{3}{4} M_{\tilde{D}}\right)$ for $\tilde{t}_{1}\left(\tilde{b}_{1}\right)$ decays, and $A_{b}\left(Q=m_{\tilde{q}_{1} O S}\right)=A_{t} \equiv A$ for $\tilde{q}_{1}$ decay, for simplicity. Moreover, we fix $\mathrm{M}=400 \mathrm{GeV}$ (i.e. $m_{\tilde{g}}=1065 \mathrm{GeV}$ ) and $m_{A}=150 \mathrm{GeV}$. Thus we have $M_{\tilde{Q}}(\tilde{t}), \mathrm{A}, \mu$ and $\tan \beta$ as free parameters. In the plots we impose the theoretical and experimental constraints [3].
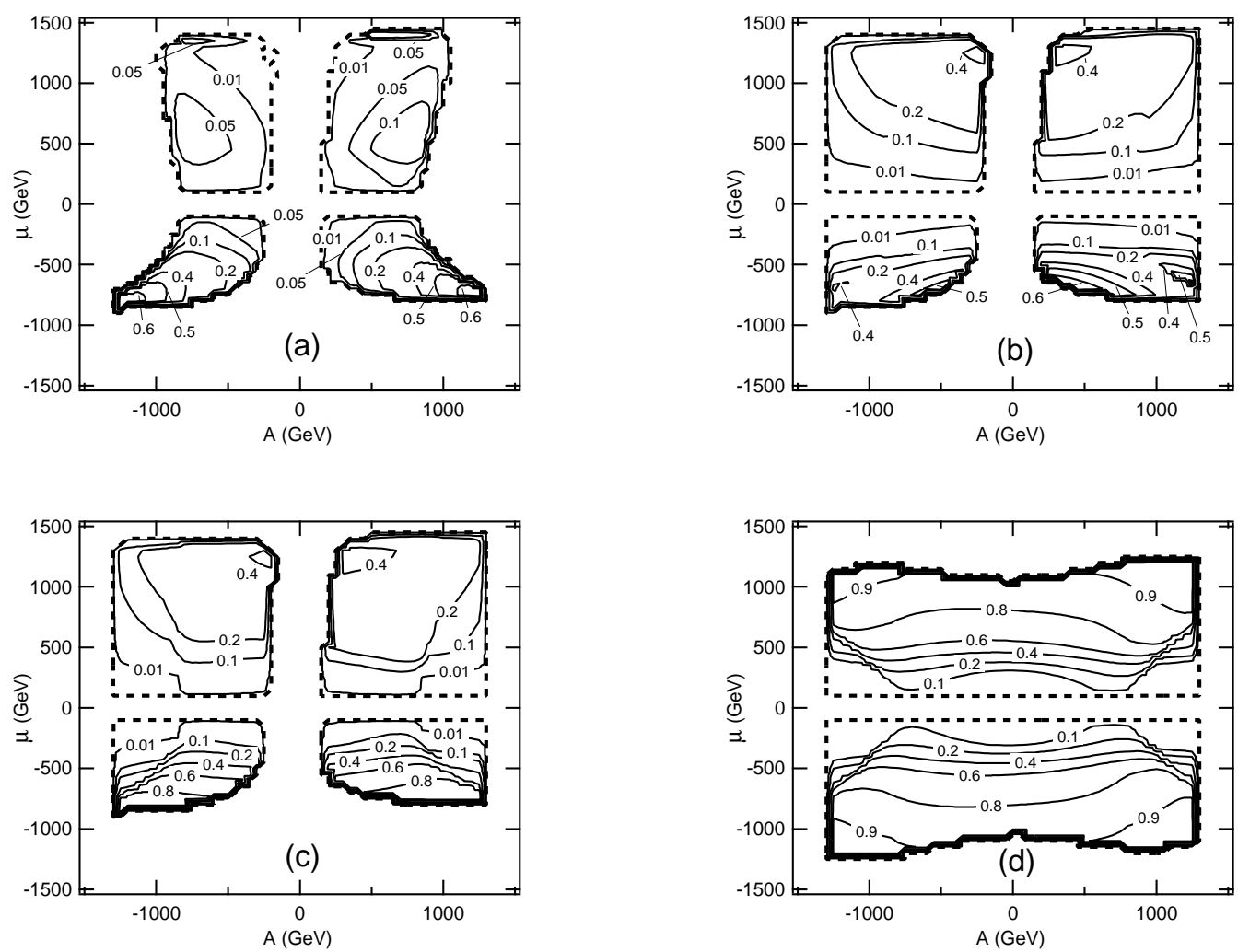

Figure 1: Contours of the branching ratios at the RG-improved tree-level in the A$\mu$ plane for $\tan \beta=30$, and $M_{\tilde{Q}}(\tilde{t})=\frac{3}{4} M_{\tilde{U}}=\frac{3}{2} M_{\tilde{D}}=600 \mathrm{GeV} ;\left(\right.$ a) $B\left(\tilde{t}_{1} \rightarrow \tilde{b}_{1}+H^{+}\right)$, (b) $B\left(\tilde{t}_{1} \rightarrow \tilde{b}_{1}+W^{+}\right)$, and (c) $B\left(\tilde{t}_{1} \rightarrow \tilde{b}_{1}+\left(H^{+}, W^{+}\right)\right)$. The regions outside of the dashed loops are excluded by the kinematics and/or the constraints given in the text. Contours of the corresponding branching ratio $B\left(\tilde{t}_{1} \rightarrow \tilde{b}_{1}+\left(H^{+}, W^{+}\right)\right)$at the naive tree-level are shown in Fig.d.

In Fig.1 we plot in the A- $\mu$ plane the contours of the $\tilde{t}_{1}$ decay branching ratios of the Higgs boson mode, the gauge boson mode, and the total bosonic modes $B\left(\tilde{t}_{1} \rightarrow\right.$ $\left.\tilde{b}_{1}+\left(H^{+}, W^{+}\right)\right) \equiv B\left(\tilde{t}_{1} \rightarrow \tilde{b}_{1}+H^{+}\right)+B\left(\tilde{t}_{1} \rightarrow \tilde{b}_{1}+W^{+}\right)$at the RG-improved tree-level. We show also those of the corresponding branching ratio $B\left(\tilde{t}_{1} \rightarrow \tilde{b}_{1}+\left(H^{+}, W^{+}\right)\right)$at the naive (unimproved) tree-level, where all input parameters are bare ones (see Eqs.(225)). We see that the $\tilde{t}_{1}$ decays into bosons are dominant in a large region of the A- $\mu$ plane, especially for large $|A|$ and/or $|\mu|$, as we expected. Comparing Fig.1.c with Fig.1.d we find that the effect of running of the quark and squark parameters $\left(m_{q}(Q), A_{q}(Q), M_{\tilde{Q}, \tilde{U}, \tilde{D}}(Q)\right)$ 
is quite dramatic. For $\tilde{b}_{1}$ decays we have obtained similar results to those for the $\tilde{t}_{1}$ decays [3].

In Fig.3 of Ref. [3] we show the individual branching ratios of the $\tilde{t}_{1}$ and $\tilde{b}_{1}$ decays as a function of $\tan \beta$ for $\left(A, \mu, M_{\tilde{Q}}(\tilde{t})\right)=(-800,-700,600) \mathrm{GeV}$ and $(800,800,600) \mathrm{GeV}$, respectively. We find that the branching ratios of the $\tilde{t}_{1}$ decays into bosons increase with increasing $\tan \beta$ and become dominant for large $\tan \beta(\gtrsim 20)$, while the $\tilde{b}_{1}$ decays into bosons are dominant in the entire range of $\tan \beta$ shown, as expected [3].

We find that the dominance of the bosonic modes is fairly insensitive to the choice of the values of $m_{A}, \mathrm{M}$, and the ratio $A_{b}(Q) / A_{t}$.

In conclusion, we have shown that the $\tilde{t}_{1}$ and $\tilde{b}_{1}$ decays into Higgs or gauge bosons can be dominant in a fairly wide MSSM parameter region with large mass difference between $\tilde{t}_{1}$ and $\tilde{b}_{1}$, large $\left|A_{t, b}\right|$ and/or $|\mu|$, and large $m_{\tilde{g}}$ (and large $\tan \beta$ for the $\tilde{t}_{1}$ decay). Compared to the conventional fermionic decays these bosonic decays can have significantly different decay distributions [3]. We have also shown that the effect of the SUSY-QCD running of the quark and squark parameters on the $\tilde{t}_{1}$ and $\tilde{b}_{1}$ decays is quite dramatic. These could

have an important impact on the searches for $\tilde{t}_{1}$ and $\tilde{b}_{1}$ and on the determination of the MSSM parameters at future colliders.

\section{Acknowledgments}

The author thanks A. Bartl very much for enjoyable collaborataion [3].

\section{References}

[1] A. Bartl, W. Majerotto, and W. Porod, Z. Phys. C 64 (1994) 499; C 68 (1995) 518 $(\mathrm{E})$.

[2] For example, R. Dermisek, a talk in this Conference.

[3] K. Hidaka and A. Bartl, Phys. Lett. B501 (2001) 78; hep-ph/0012021.

[4] A. Bartl, H. Eberl, K. Hidaka, S. Kraml, W. Majerotto, W. Porod, and Y. Yamada, Phys. Rev. D 59 (1999) 115007.

[5] H. Eberl, K. Hidaka, S. Kraml, W. Majerotto, and Y. Yamada, Phys. Rev. D62 (2000) 055006. 